\title{
Model Ensemble-Based Prognostic Framework for Fatigue Crack Growth Prediction
}

\author{
Hoang-Phuong Nguyen, Enrico Zio \\ Chair on System Science and the Energetic Challenge \\ CentraleSupélec, Université Paris-Saclay \\ 91192 Gif-sur-Yvette, France \\ e-mail: hoang-phuong.nguyen@centralesupelec.fr, \\ enrico.zio@centralesupelec.fr
}

\author{
Jie Liu \\ School of Reliability and Systems Engineering \\ Beihang University \\ 37 Xueyuan Road, Haidian, Beijing, China \\ e-mail: liujie805@buaa.edu.cn
}

\begin{abstract}
The demand for online fatigue crack growth prognosis has recently increased in industry in order to prevent severe unexpected failures in equipment operated in evolving conditions where static models may no longer perform well. To address this issue, a robust prognostic framework is presented in this paper to assess the reliability of deteriorating equipment due to fatigue crack growth. In this framework, a new model ensemble methodology that integrates multiple stochastic crack growth models based on the quadratic bestworst weighted voting (QBWWV) is proposed for predicting the remaining useful life (RUL) of equipment. To validate the effectiveness of the proposed framework, a case study concerning fatigue crack growth is demonstrated. The results indicate that the proposed prognostic framework outperforms single crack growth models in terms of prediction accuracy under evolving operating conditions.
\end{abstract}

Keywords-Prognostics and Health Management (PHM); remaining useful life (RUL); recursive Bayesian; dynamic ensemble; fatigue crack growth

\section{INTRODUCTION}

With the fast-pace advancement of technology, industrial systems are being given opportunities to become more and more efficient, complex and faster [1]. For this, the development of reliability and health management strategies of components and systems is playing a vital role for industry. These strategies aim at early detecting the degradation of engineering components, diagnosing their faults, proactively predicting their future health state evolution and remaining useful life (RUL), based on the operational and historical failure information, enabling optimal maintenance decisions before the breakdown of the components. In general, the reliability of equipment frequently decreases due to gradual growing degradations, such as fatigue crack, delamination processes, corrosion, and eventually leading to failure. Among them, fatigue crack growth is one of the most common degradation, that drives the components and systems to critical failures in several major industries, including manufacturing [2], [3], automotive [4], [5], aerospace [6], [7], etc. To prevent such unanticipated failures, which can cause unexpected interruptions in production, the development of prognosis systems for addressing fatigue crack growth is crucial.
One of the main concerns of fatigue crack propagation is assessing the rate of crack growth, which is often done with empirical models using condition monitoring data [8]-[10]. This enables online reliability assessment, whereby the reliability of the equipment can be estimated timedependently based on the prognostic results and the prior assessment, with reduced uncertainty. In [8], a failure prognostic scheme for fatigue crack growth prediction was introduced, which employed a stochastic crack growth model and a Bayesian technique to dynamically update the degradation state of equipment from a sequence of monitored measurements. In the field of model-based prognostics, recursive Bayesian-based schemes have been extensively utilized due to the possibility of updating the posterior distribution of the degradation states as more data is collected during the course of time. In [9], a Bayesianbased prognostic approach was presented to online-estimate the stress intensive range of the degradation model, at each load cycle until fracture. By exploiting a combination of a non-linear degradation model and a Bayesian variant, [10] proposed a comprehensive architecture for failure detection, isolation and prognosis for detecting abnormal symptoms and online predict the crack depth evolution of the UH-60 planetary carrier plate.

However, the performance of model-based prognostic frameworks for fatigue crack growth greatly depends on the adopted physics-of-failure model. To address this issue, the demand of finding out an optimal modelling framework, especially for a specific degradation process under timevarying operation conditions, is crucial. In recent decades, several studies on fatigue crack growth models have been extensively investigated and developed [11]-[15]. In [12], a comprehensive comparison of stochastic models for fatigue crack growth, including the Markov chain model, the Yang's power law-based model, and a polynomial model, was carried out. The results showed that each degradation model has its condition and range of applicability, which means that each model is appropriate only to specific degradation processes. To the knowledge of the authors, there is no general consensus on a reliably unique prognostic model for fatigue crack growth under different degradation processes. Recently, hybrid and multi-degradation models ensembles have attracted the attention of industrial practitioners and researcher due to higher accuracy and better generalization 
capability than individual degradation models [16], [17]. The basic idea behind these empirical frameworks is to exploit the diversity of different degradation models, which can offer complementary information about the degradation states to be estimated. For example, in an application of Lithium-ion battery prognostics, an interacting multiple model particle filter (IMMF) was presented to combine the estimations from three battery capacity degradation models [17]. The results experimentally showed that the ensemble method yields a better generalization performance over a single model in terms of smaller estimation errors and more precise predictions.

In this paper, a prognostic framework based on a recursive Bayesian technique and a dynamic ensemble is proposed for assessing the reliability of deteriorating equipment due to fatigue crack growth. Diversity and accuracy are two key factors deciding the generalization performance of an ensemble framework. However, it is difficult constructing a good ensemble by balancing these two factors, because increasing diversity maybe at the cost of decreasing accuracy and vice versa. To address this issue, we introduce a new ensemble weight coefficient based on minimizing the historical prediction errors of each physical model in the ensemble and combining with the quadratic best-worst weighted voting (QBWWV) strategy. Finally, a case study concerning fatigue crack growth is carried out to demonstrate the improved performance of the proposed framework and the results are compared with those obtained by applying single degradation models.

The rest of this paper is organized as follows. Section II introduces the degradation models for fatigue crack growth and details the proposed prognostic framework. Section III describes the illustrative case study of fatigue crack growth and shows the experimental results of the proposed framework. Finally, Section IV concludes the study.

\section{PROPOSED PROGNOSTIC FRAMEWORK}

\section{A. Recursive Bayesian Technique for Degradation State Estimations}

Consider a state-space model given by:

$$
\begin{gathered}
x_{t}=f_{t}\left(x_{t-1}, \omega_{t-1}\right) \\
z_{t}=g_{t}\left(x_{t}, v_{t}\right),
\end{gathered}
$$

where $x_{t}$ and $z_{t}$ denote the degradation state and the measurement at the inspection time $t\{t \in \mathrm{N}\}$, respectively; $f_{t}$ is the state transition function and $g_{t}$ is the measurement function; $\omega_{t-1}$ and $v_{t}$ are independent identically distributed (i.i.d.) state noise and measurement noise sequences, respectively. This represents a first-order Markov process with independent degradation increments, where the current degradation state $x_{t}$ depends only on the previous state $x_{t-1}$, and not on the states at previous times. The system state $x_{t}$ at time $t$ can be estimated by constructing its posterior probability density function (pdf), $p\left(x_{t} \mid z_{1: t}\right)$ via the two following steps, namely prediction and update.

In the prediction step, both the previous state estimation $x_{t-1}$ and the state transition model $f_{t}$ are employed to estimate the prior distribution of the current state $x_{t}$ via the Chapman-Kolmogorov equation, as follows:

$$
\begin{aligned}
p\left(x_{t} \mid z_{1: t-1}\right) & =\int p\left(x_{t} \mid x_{t-1}, z_{1: t-1}\right) p\left(x_{t-1} \mid z_{1: t-1}\right) d x_{t-1} \\
& =\int p\left(x_{t} \mid x_{t-1}\right) p\left(x_{t-1} \mid z_{1: t-1}\right) d x_{t-1}
\end{aligned}
$$

where $p\left(x_{t} \mid x_{t-1}\right)$ is the conditional probability distribution defined by the state model in (1). In this work, the initial distribution $p\left(x_{0} \mid z_{0}\right)=p\left(x_{0}\right)$ is assumed to be available and is known as the prior. In the update step, as the new measurement $z_{t}$ is collected, the posterior distribution $p\left(x_{t} \mid z_{1: t}\right)$ can be estimated by using Bayes' theorem, as follows:

$$
p\left(x_{t} \mid z_{1: t}\right)=\frac{p\left(x_{t} \mid z_{1: t-1}\right) p\left(z_{t} \mid x_{t}\right)}{p\left(z_{t} \mid z_{1: t-1}\right)},
$$

where $p\left(z_{t} \mid x_{t}\right)$ is the likelihood function defined by the measurement model in (2) and $p\left(z_{t} \mid z_{1: t-1}\right)$ is a normalizing constant, which is defined by:

$$
p\left(z_{t} \mid z_{1: t-1}\right)=\int p\left(x_{t} \mid z_{1: t-1}\right) p\left(z_{t} \mid x_{t}\right) d x_{t}
$$

\section{B. Fatigue Crack Growth Models}

As mentioned above, diversity is an important aspect that needs to be considered in the design of an ensemble framework. The ideal situation occurs when an ensemble is composed of a set of models with uncorrelated errors, where diversity can be fully exploited. In this paper, four stochastic degradation models are selected for fatigue crack growth prediction, including Paris-Erdogan, polynomial, global function-based, and curve fitting function-based models.

1) Paris-Erdogan model:The relation between the crack growth rate $\frac{d x}{d N}$ and the Irwin's stress intensity factor $\Delta K$ [13] is defined as follows:

$$
\frac{d x}{d N}=C(\Delta K)^{m}
$$

where $x$ is the crack length, $C$ and $m$ are material constants, and $N$ is the number of fatigue load cycles. In this model, the stress intensity factor $\Delta K$ is defined as follows [13]: 


$$
\Delta K=\Delta \sigma \sqrt{\pi x},
$$

where $\Delta \sigma$ is the cyclic stress amplitude.

To take into consideration the statistical variability of the crack growth rate in practice, a stochastic variant of the Paris-Erdogan model is introduced by injecting a process intrinsic stochasticity as follows [14]:

$$
\frac{d x}{d N}=e^{\omega} C(\Delta K)^{m}
$$

where $\omega \sim N\left(0, \sigma_{\omega}^{2}\right)$ is an additional white Gaussian noise. For a sufficiently small $\Delta t$, the Markov chain state-space model of the degradation state $x$ in (8) can be discretized as follows:

$$
x_{t}=x_{t-1}+e^{\omega} C(\Delta K)^{m} \Delta t,
$$

2) Polynomial model: In [12], [15], a polynomial function-based model was introduced for addressing the mismatch of power function-based crack growth models, i.e. Paris-Erdogan model, with the median crack growth curve, given by:

$$
\frac{d x}{d N}=e^{\omega}\left(p_{0}+p_{1} x+p_{2} x^{2}\right)
$$

where $p_{i}, i=0, \ldots 2$ are polynomial constants. The polynomial model was also reported to yield the best fit in the linear least square stage of the degradation process [15], [16]. The Markov process representation for a polynomial function-based crack growth model can be defined as follows:

$$
x_{t}=x_{t-1}+e^{\omega}\left(p_{0}+p_{1} x+p_{2} x^{2}\right) \Delta t
$$

3) Global function: Considering that the nature of structural fatigue crack growth is generally determined by multiple factors, such as material properties and equipment geometry, which always exhibit some degree of uncertainty on the degradation process, a global function was introduced by reformulating the stress intensity factor, given by [11]:

$$
\Delta K=h(x) \Delta \sigma \sqrt{\pi x},
$$

where $h(x)$ denotes the geometric factor of fatigue crack, defined as follows:

$$
h(x)=\alpha_{0}+\alpha_{1} \frac{x}{w}+\alpha_{2}\left(\frac{x}{w}\right)^{2}+\alpha_{3}\left(\frac{x}{w}\right)^{3}
$$

where $\alpha_{i}, i=0, \ldots, 3$ are geometric coefficients and $w$ is the specimen width. The global function-based crack growth model can be further discretized as follows:

$$
x_{t}=x_{t-1}+e^{\omega} C(h(x) \Delta \sigma \sqrt{\pi x})^{m} \Delta t
$$

4) Curve fitting function: Still in [11], the authors presented another empirical crack growth model based on a curve fitting technique, which outperformed the conventional models, such as the power function and the polynomial function, in terms of higher prediction accuracy and lower computational cost. The curve fitting functionbased model is given by:

$$
\frac{d x}{d N}=e^{\omega}\left(\frac{1}{C_{1} x^{m}+C_{2}}\right),
$$

where $C_{1}, C_{2}, m$ denote the model constants.

The discretized Markov process representation for the model can be defined as follows:

$$
x_{t}=x_{t-1}+e^{\omega}\left(\frac{1}{C_{1} x^{m}+C_{2}}\right)(\Delta K)^{m} \Delta t
$$

\section{Dynamic-Weighted Ensemble for Prediction}

In this section, a selective ensemble based on the QBWWV strategy is presented to dynamically find the best combination of multiple crack growth models with respect to their estimation performances. The proposed algorithm consists of the following three steps:

1) Step 1: At the load cycle time $t$, the current degradation state given by each model is updated when new measurement is available by using the recursive Bayesian algorithm described in Section II.A.

2) Step 2: The ensemble weight of each single model, at time $t$, is then updated based on the QBWWV and their estimation errors for the last cycles as follows:

$$
w_{t}^{i}=\left(\frac{\varphi_{t}^{\max }-\varphi_{t}^{i}}{\varphi_{t}^{\max }-\varphi_{t}^{\min }}\right)^{2},
$$

where $w_{t}^{i}$ and $\varphi_{t}^{i}$ are the dynamic weight and estimation error coefficient of the $i$ th model at time $t$, respectively; $\varphi_{t}^{\max }$ and $\varphi_{t}^{\min }$ are the maximum and minimum error coefficients of all models at time $t$. In this scheme, the model with the highest estimation error will be removed from the ensemble. The estimation error coefficient $\varphi_{t}^{i}$ is defined as follows: 


$$
\varphi_{t}^{i}=\frac{1}{\delta} \sum_{k=t-\delta}^{t}\left(z_{k}-\hat{x}_{k}^{i}\right)^{2}
$$

where $\delta$ is the time horizon ( $\delta=50$ load cycles in the case study that follows) and $\hat{x}_{k}^{i}$ is the estimated degradation state of the $i$ th model at time $k$.

3) Step 3: Once the ensemble weights for all models at the current time $t$ are updated, the estimated degradation state of the ensemble is computed as follows:

$$
\tilde{x}_{t}=\sum_{i=1}^{N_{M}}\left(\hat{x}_{t}^{i} \times \widetilde{w}_{t}^{i}\right)
$$

where $\tilde{x}_{t}$ is the ensemble-predicted state at the load cycle $t$, $\widetilde{w}_{t}^{i}$ is the normalized weight for each single model and $N_{M}$ is the number of degradation models ( $N_{M}=4$ in this study).

\section{RESULTS AND DISCUSSION}

The proposed prognostic framework is applied to a case study of fatigue crack growth, which has been carried out with an initial length $x_{0}=10^{-4} \mathrm{~mm}$ and a failure threshold at $d^{*}=60 \mathrm{~mm}$. The common Paris-Erdogan model in (9) is adopted for describing the evolution of the crack depth with a total number of fatigue crack load cycles, $N=2000$ cycles. The parameters of the state equation (9) are $C=0.1$ and $m=$ 1.3 , whereas the state and measurement noise variances are $\sigma_{\omega}^{2}=0.49$ and $\sigma_{v}^{2}=0.16$, respectively [8]. Fig. 1 shows the simulated crack depth evolution, if no preventive maintenance is undertaken.

When a new monitored measurement is available, the degradation state of each individual model is updated at the current load cycle, via the recursive Bayesian technique. Their ensemble weights are also updated based on the estimation errors for the last cycles, as shown in Fig. 2. The results show that the four crack growth models yield similar performances when the crack size is sufficiently small, resulting in equivalent weights during the first 1000 cycles. However, as the crack depth exponentially increases, the dynamic weights are varying and discriminate between different models due to the evolving operation conditions.

The results of degradation state estimations and RUL predictions are shown in Figs. 3 and 4. As can be seen in Fig. 4 , when the crack depth rapidly increases in the later 1000 cycles, the proposed ensemble approach can achieve performance superior to the single models in predicting the health state evolution and the RUL of equipment. In addition, as more and more measurements are collected, the prediction of the proposed ensemble approach continues showing better performance, yielding the closest predictions to the groundtruth RUL. Table I shows that the proposed ensemble framework outperforms the individual crack growth models, yielding an estimation accuracy of 0.1112 in terms of the mean square error (MSE), calculated between the true degradation state and the estimation of each crack growth model.

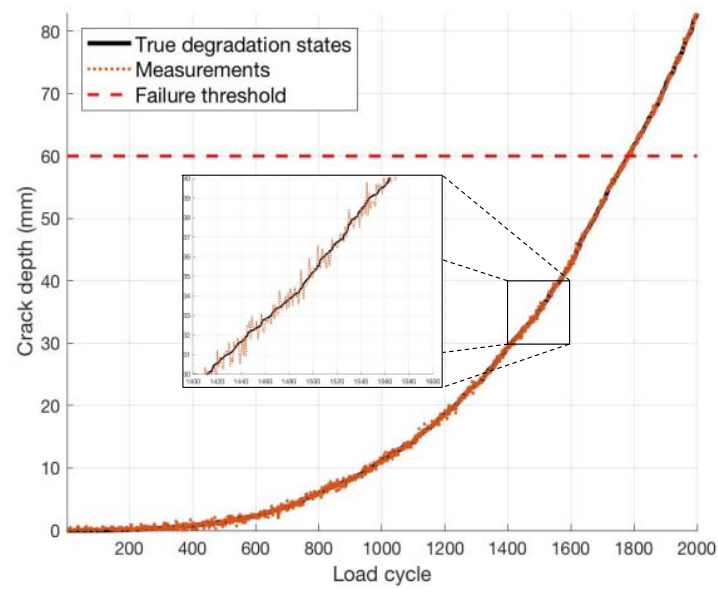

Figure 1. Simulated crack depth evolution.

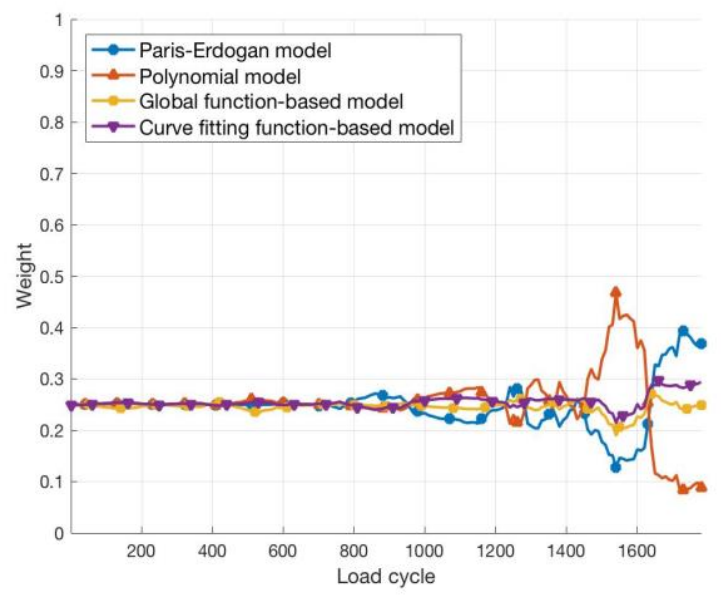

Figure 2. Dynamic ensemble weights of the individual models.

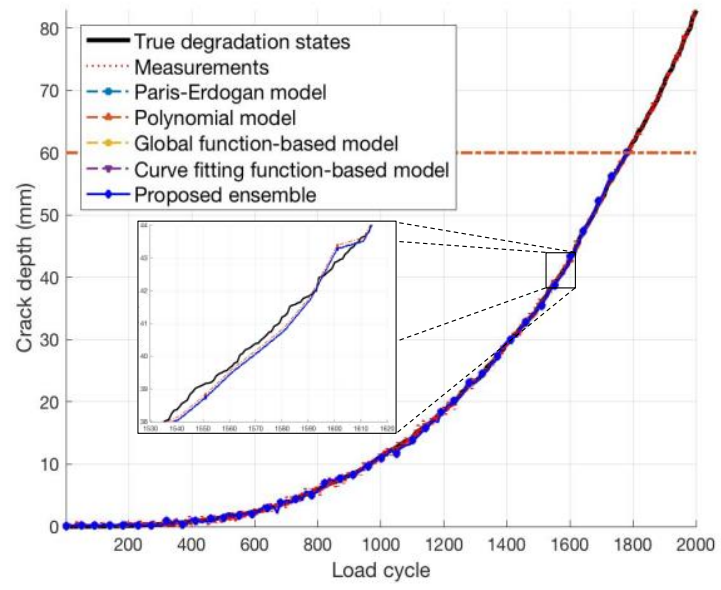

Figure 3. Degradation state estimations based on the individual models and the proposed ensemble. 


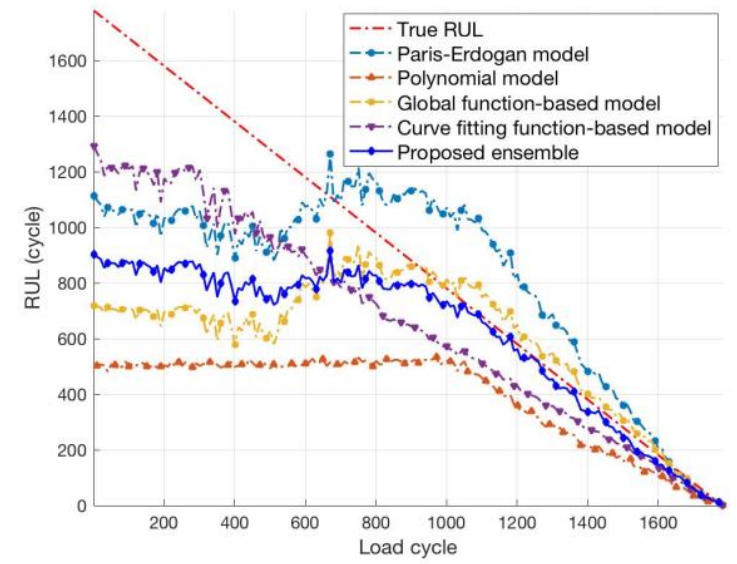

Figure 4. RUL predictions based on the individual models and the proposed ensemble.

TABLE I. MSE RESULTS FOR DEGRADATION STATE ESTIMATIONS.

\begin{tabular}{cccccc}
\hline \multirow{2}{*}{ MSE } & $\begin{array}{c}\text { Paris- } \\
\text { Erdogan }\end{array}$ & Polynomial & $\begin{array}{c}\text { Global } \\
\text { function }\end{array}$ & $\begin{array}{c}\text { Curve- } \\
\text { fitting }\end{array}$ & $\begin{array}{c}\text { Proposed } \\
\text { ensemble }\end{array}$ \\
\cline { 2 - 6 } & 0.1171 & 0.1142 & 0.1149 & 0.1126 & 0.1112 \\
\hline
\end{tabular}

IV. CONCLUSIONS

This paper proposes an online prognostic framework for predicting the degradation states and the RUL of deteriorating equipment due to fatigue crack growth. Although several degradation models have been investigated for fatigue crack growth prognosis in the literature, there is no general consensus on a reliably unique crack growth model for different degradation processes. To address this issue, a dynamic-weighted ensemble of multiple degradation models is presented. The key idea of the proposed approach is to utilize the QBWWV strategy and a dynamic weight vector, which is updated at each load cycle when the new measurements become available, for evaluating individual degradation models performance with respect to their estimation errors on previous cycles. Simulation results show that the proposed prognostic framework can yield a satisfactory performance under evolving operating conditions, and outperforms individual models for fatigue crack growth in terms of prediction accuracy.

\section{REFERENCES}

[1] E. Zio, "Some Challenges and Opportunities in Reliability Engineering," IEEE Trans. Reliab., vol. PP, no. 99, pp. 1769-1782, 2016.
[2] S. Eckmann and C. Schweizer, "Characterization of fatigue crack growth, damage mechanisms and damage evolution of the nickelbased superalloys MAR-M247 CC (HIP) and CM-247 LC under thermomechanical fatigue loading using in situ optical microscopy," Int. J. Fatigue, vol. 99, pp. 235-241, 2017.

[3] M. Kamaya, "Fatigue crack tolerance design for stainless steel by crack growth analysis," Eng. Fract. Mech., vol. 177, pp. 14-32, 2017.

[4] H. K. Yang, V. Doquet, and Z. F. Zhang, "Fatigue crack growth in two TWIP steels with different stacking fault energies," Int. J. Fatigue, vol. 98, pp. 247-258, 2017.

[5] J. Mayén, A. Abúndez, I. Pereyra, J. Colín, A. Blanco, and S. Serna, "Comparative analysis of the fatigue short crack growth on Al 6061T6 alloy by the exponential crack growth equation and a proposed empirical model," Eng. Fract. Mech., vol. 177, pp. 203-217, 2017.

[6] M. A. Haile, J. C. Riddick, and A. H. Assefa, "Robust Particle Filters for Fatigue Crack Growth Estimation in Rotorcraft Structures," IEEE Transactions on Reliability, vol. 65, no. 3. pp. 1438-1448, 2016.

[7] M. Haile, T.-K. Chen, M. Shiao, and D. Le, "Crack growth behavior in preloaded metallic nested-angle plates under flight load spectrum," in Experimental and Applied Mechanics, Volume 6: Proceedings of the 2011 Annual Conference on Experimental and Applied Mechanics, T. Proulx, Ed. New York, NY: Springer New York, 2011, pp. 3-11.

[8] F. Cadini, E. Zio, and D. Avram, "Model-based Monte Carlo state estimation for condition-based component replacement," Reliab. Eng. Syst. Saf., vol. 94, no. 3, pp. 752-758, Mar. 2009.

[9] B. A. Zárate, J. M. Caicedo, J. Yu, and P. Ziehl, "Bayesian model updating and prognosis of fatigue crack growth," Eng. Struct., vol. 45, pp. 53-61, Dec. 2012.

[10] M. E. Orchard, "A Particle Filtering-based Framework for Online Fault Diagnosis and Failure Prognosis," Georgia Institute of Technology, 2007.

[11] H. Salimi, M. Pourgol-Mohammad, and S. Kiad, "Assessment of Stochastic Fatigue Failures Based On Deterministic Functions," in 13th International conference on Probabilistic Safety Assessment and Management (PSAM 13), 2016.

[12] W. F. Wu and C. C. Ni, "Probabilistic models of fatigue crack propagation and their experimental verification," Probabilistic Eng. Mech., vol. 19, no. 3, pp. 247-257, Jul. 2004.

[13] G. R. Irwin, "Analysis of Stresses and Strains Near the End of a Crack Traversing a Plate," J. Appl. Mech., 1957.

[14] E. Myötyri, U. Pulkkinen, and K. Simola, "Application of stochastic filtering for lifetime prediction," Reliab. Eng. Syst. Saf., vol. 91, no. 2, pp. 200-208, Feb. 2006.

[15] C. C. Ni, "Formulation of a Polynomial Stochastic Fatigue Crack Growth Model," Adv. Mater. Res., vol. 909, pp. 467-471, 2014.

[16] Y. Xing, E. W. M. Ma, K.-L. Tsui, and M. Pecht, "An ensemble model for predicting the remaining useful performance of lithium-ion batteries," Microelectron. Reliab., vol. 53, no. 6, pp. 811-820, Jun. 2013.

[17] X. Su, S. Wang, M. Pecht, L. Zhao, and Z. Ye, "Interacting multiple model particle filter for prognostics of lithium-ion batteries," Microelectron. Reliab., vol. 70, pp. 59-69, 2017. 